\title{
INAVA promotes aggressiveness of papillary thyroid cancer by upregulating MMP9 expression
}

Hongyu Guan ${ }^{1 \dagger}$, Yan Guo ${ }^{1 \dagger}$, Liehua Liu' ${ }^{1 \dagger}$, Runyi Ye ${ }^{2}$, Weiwei Liang ${ }^{1}$, Hai Li ${ }^{1}$, Haipeng Xiao ${ }^{1}$ and Yanbing Li ${ }^{*}$

\begin{abstract}
Background: Innate immunity activator (INAVA) has been shown to be elevated in lung adenocarcinoma. However, its expression pattern and function in papillary thyroid cancer (PTC) are unknown. This study aimed to identify the clinical, biological, and mechanistic impacts of INAVA on PTC.

Methods: Using The Cancer Genome Atlas dataset, real time PCR, and immunohistochemistry, the expression of INAVA in PTC was analyzed. Gain- and loss-of-function assays were performed to investigate the role of INAVA in PTC cell invasion, migration, and metastasis. We explored the molecular mechanisms underlying the roles of INAVA in PTC cells using transcriptome resequencing, real time PCR, western blotting and immunohistochemistry.

Results: We found that INAVA expression was significantly upregulated in PTC and was significantly associated with lymph node metastasis. Loss- and gain-of-function experiments demonstrated that INAVA promoted the aggressive phenotype of PTC cells in vitro and in vivo. Mechanistic study suggested that upregulation of INAVA resulted in elevated fibroblast growth factor 1 (FGF1), which in turn increased the expression level of matrix metalloproteinases 9 (MMP9). We further identified that the level of INAVA was positively correlated with the levels of FGF1 and MMP9 in clinical PTC specimens.
\end{abstract}

Conclusion: These data establish a novel role for INAVA in promoting PTC progression and suggest that INAVA may represent a therapeutic target for the disease.

Keywords: INAVA, Papillary thyroid cancer, Invasion, MMP9, FGF1

\section{Background}

Thyroid cancer is the most common type of endocrinerelated malignancy and shows rapidly increasing incidences during the past decades [1]. Papillary thyroid cancer (PTC) is the most common form of thyroid cancer, representing $70-80 \%$ of all thyroid cancer [2]. In most cases, patients with PTC follow an indolent course and have excellent prognosis [3]. However, extrathyroidal extension and distant metastasis are extremely important risk factors for PTC and are still major clinical challenges

\footnotetext{
*Correspondence: easd04lyb@126.com

${ }^{\dagger}$ Hongyu Guan, Yan Guo, and Liehua Liu contributed equally to this work

1 Department of Endocrinology and Diabetes Center, The First

Affiliated Hospital of Sun Yat-sen University, 58 Zhongshan Road II,

Guangzhou 510080, Guangdong, China

Full list of author information is available at the end of the article
}

$[4,5]$. Thus, a good understanding of the molecular alterations responsible for aggressive behavior of PTC is still imperative.

Tumor metastasis is a multistep process during which a subset of cancer cells disseminate from the site of a primary tumor and establish secondary tumors at distant sites [6]. Matrix metalloproteinases (MMPs) are proteolytic enzymes responsible for remodeling extracellular matrix (ECM) in many physiological and pathological processes [7]. Moreover, besides its role in degradation of ECM molecules, MMPs play important roles in multiple aspects of cancer progression, including tumor growth, almost all metastatic steps, and angiogenesis $[8,9]$. MMP9, one of the best characterized members in MMP family, is well known to degrade ECM and is closely related to the cancer invasion and 
metastasis [10]. In PTC, Ahmed et al. showed that silencing of FoxM1 inhibited migration and invasion of PTC cells via decreasing the expression levels of MMP9 and MMP2 [11]. Moreover, the expression level of arachidonate 5-lipoxygenase (ALOX5) was upregulated in PTC and it promoted PTC cell invasion through increased secretion of MMP9 [12].

Innate immunity activator (INAVA, also named chromosome 1 open reading frame 106) is a protein coding gene known to be a risk locus for Crohn's disease $[13,14]$. Notably, a global analysis of chromosome 1 genes among patients with lung adenocarcinoma found that INAVA was upregulated at four stages of lung adenocarcinoma, implicating that INAVA may play important roles in cancer development and progression [15]. Nevertheless, the role of INAVA has not been investigated in malignant diseases. Here, we investigated the expression pattern of INAVA in human PTC and attempted to explore the molecular mechanisms mediating the possible oncogenic functions of INAVA.

\section{Methods}

\section{Cell lines and reagents}

The human PTC cell line K1 was purchased from the European Collection of Cell Cultures (ECACC, Salisbury, UK), and the TPC1 cell line was provided by Prof. Haixia Guan (China Medical University, China). All cell lines were authenticated by short tandem repeat (STR) DNA profiling and were verified to be mycoplasma-free. The cells were cultured in Dulbecco's modified Eagle's medium (DMEM) (Gibco, Grand Island, NY) containing $10 \%$ fetal bovine serum (FBS; Gibco), $100 \mathrm{U} / \mathrm{mL}$ penicillin (Gibco), and $100 \mathrm{mg} / \mathrm{mL}$ streptomycin (Gibco) at $37^{\circ} \mathrm{C}$ in a $5 \% \mathrm{CO}_{2}$ humidified incubator.

\section{Patients and tissue specimens}

The 112 cases of paraffin-embedded PTC and 16 pairs of fresh PTC and their adjacent noncancerous thyroid tissues were histopathologically and clinically diagnosed at the First Affiliated Hospital of Sun Yat-sen University from 2011 to 2016. The 16 pairs of fresh tissues were collected from 16 patients, and then were frozen and stored in liquid nitrogen until used. The pathology of all specimens was confirmed by two pathologists. Informed consents from patients and ethics approval from the Institutional Research Ethics Committee were obtained.

\section{Immunohistochemistry (IHC)}

Paraffin sections were deparaffinized with xylene, rehydrated with alcohol, subjected to antigen retrieval in sodium citrate buffer $(10 \mathrm{mM}, \mathrm{pH} 6.0)$ and blocked in PBS containing $1 \%$ (wt/vol) bovine serum albumin (BSA; Sigma-Aldrich, St. Louis, MO). Then the sections were incubated with primary antibodies against INAVA and fibroblast growth factor 1 (FGF1) (Abcam, Cambridge, MA), MMP9 (Cell Signaling Technology). The IHC staining was evaluated as described in our previous study [16]. Briefly, the intensity of staining was scored as 0 (no staining), 1 (weak staining = light yellow), 2 (moderate staining = yellow brown), or 3 (strong staining=brown). The proportion of tumor cells was scored as 0 (no positive tumor cells), 1 ( $<10 \%$ positive tumor cells), 2 (10-50\% positive tumor cells), or 3 ( $>50 \%$ positive tumor cells). The staining index (SI) was calculated by multiplying the intensity and proportion score. An SI score of $\geq 4$ was regarded as tumors with high expression and $\leq 3$ as tumors with low expression.

\section{Vectors and retroviral infection}

INAVA construct was generated by sub-cloning PCRamplified full-length human INAVA cDNA into pQCXIP (Clontech, Mountain View, CA). The pRS-INAVA-shRNAs (TR306059) were purchased from OriGene (Rockville, MD). These plasmids were transfected into PT67 cells (Clontech) using Lipofectamine 3000 (Invitrogen, San Diego, CA). After transfection, the PT67 cells were cultured for $48 \mathrm{~h}$. Then, the supernatant was harvested, passed through a $0.45-\mu \mathrm{m}$ filter and incubated with the indicated cells along with polybrene $(8 \mu \mathrm{g} / \mathrm{mL})$. Subsequently, stable cell lines were selected with $0.5 \mu \mathrm{g} / \mathrm{mL}$ of puromycin for 2 weeks [17].

\section{RNA extraction and real-time reverse}

\section{transcription-polymerase chain reaction (RT-PCR)}

Total RNA was extracted using TRIzol (Invitrogen). RT was carried out with the Moloney murine leukemia virus reverse transcriptase (MMLV-RT) (Promega, Madison, WI) using $1 \mu \mathrm{g}$ of total RNA as the template. Real-time RT-PCR detection was performed using SYBR Premix Ex Taq (TaKaRa, Dalian, China) on a 7500 system (Applied Biosystems, Carlsbad, CA). Glyceraldehyde-3-phosphate dehydrogenase (GAPDH) was used as an internal control. The following primers were used: MMP9 forward, 5'-ACGACGTCTTCCAGTACCGA-3' and reverse, 5'-TTGGTCCACCTGGTTCAACT-3'; FGF1 forward, $5^{\prime}$-TGTGGAGAGAGGTACAGCCC- $3^{\prime}$ and reverse, 5'-AAGGTGGTGATTTCCCCTTC-3'; GAPDH forward, 5'-GACTCATGACCACAGTCCATG-3' and reverse, $5^{\prime}$-AGAGGCAGGGATGATGTTCTG-3'. The relative expression levels were determined using the $2^{-\Delta \Delta \mathrm{Ct}}$ method. Each sample was analyzed three times in triplicate.

\section{Western blotting (WB) analysis}

The indicated cells were harvested and lysed using RIPA lysis buffer (Thermo Fisher Scientific, Waltham, MA). The concentration of protein was determined by 
the bicinchoninic acid assay (BCA; Thermo Fisher Scientific). Equal amounts of proteins were separated by sodium dodecyl sulfate-polyacrylamide gel electrophoresis (SDS-PAGE) gels and then transferred to the PVDF membranes (Roche, Indianapolis, IN). The membranes were blocked with 5\% nonfat milk and then incubated with the primary antibodies: anti-INAVA antibody and anti-FGF1 antibody (Abcam), anti-MMP9 antibody (Cell Signaling Technology), and anti- $\alpha$-tubulin antibody (Sigma-Aldrich). The bands were detected using enhanced chemiluminescence (ECL) detection kit (Pierce, Rockford, IL) according to the manufacturer's instructions.

\section{Transwell assay}

The invasion and migration assays were performed using Transwell chambers (Millipore, Bedford, MA) pre-coated with or without Matrigel (BD, Bedford, MA) on the upper side in 24-well plates. A total of $2 \times 10^{4}$ cells in $200 \mu \mathrm{L}$ of serum-free DMEM medium were added to the upper chamber and $500 \mu \mathrm{L}$ of DMEM medium containing $10 \%$ FBS were added to the lower chamber. Following $24 \mathrm{~h}$ incubation, the cells on the upper surface of the chambers were removed with cotton swabs. Then the migrated and invaded cells on the lower surface were stained with $0.1 \%$ crystal violet and counted under a microscope. Each sample was analyzed three times in triplicate.

\section{Wound-healing assay}

The cell migration activity was examined using woundhealing assay. Briefly, the indicated cells were seeded in 6-well plates and grown to reach 90-95\% confluence. Then the wounds were created by scratching the cell monolayer with a micropipette tip. Cells were allowed to migrate for $18 \mathrm{~h}$ under serum-reduced medium. The wounds were observed and photographed under a microscope. The Image J software (National Institutes of Health, Bethesda, Maryland) was used to determine the distance between the wound edges.

\section{In vivo metastasis assay}

For the in vivo metastasis assay, equal amounts of $\left(6 \times 10^{6}\right)$ the luciferase-expressing K1-INAVA-Luc or K1-Vector-Luc cells were injected through the tail vein of 6 weeks old BALB/c nude mice (5 mice per group). Bioluminescence imaging was performed using the Xenogen IVIS Spectrum Imaging System (Caliper Life Sciences, Hopkinton, MA). Mice were injected i.p. with $150 \mathrm{mg} / \mathrm{kg}$ luciferin $15 \mathrm{~min}$ prior to imaging. Images were taken and analyzed using Spectrum Living Image 4.0 Software (Caliper Life Sciences). Sixty days post-injection, the mice were euthanized. The lungs were excised, embedded with paraffin and then stained with hematoxylin and eosin
(HE) [18]. Microscopic evaluation was carried out using the BX51 Olympus microscope (Olympus, Tokyo, Japan) and photographs were obtained. Metastasis area was calculated using Image J software in HE stained sections.

\section{RNA sequencing}

Transcriptome resequencing was performed by the Vazyme Biotech Co., Ltd, (Nanjing, China). Briefly, the transcriptome library for sequencing was generated using VAHTSTM mRNA-seq v2 Library Prep Kit for Illumina ${ }^{\circledR}$ (Vazyme Biotech Co.) following the manufacturer's recommendations. The clustering of the index-coded samples was performed using VAHTS RNA Adapters set1/ set2 for Illumina ${ }^{\circledR}$ (Vazyme Biotech Co.) according to the manufacturer's instructions. After clustering, the libraries were sequenced on Illumina Hiseq X Ten platform using paired-end module. Then, bioinformatics analysis was performed.

\section{Accession numbers}

The transcriptome resequencing data described herein have been deposited in the Gene Expression Omnibus (GEO) database and are accessible through GEO series accession number GSE100392.

\section{Gelatin zymography analysis}

Zymography assay was performed to examine the MMP activity. In brief, indicated cells were cultured in serumfree DMEM medium for $48 \mathrm{~h}$ before the culture media were collected and centrifuged. Equal amounts of supernatants were added with equal amounts of $2 \times$ Zymogram sample buffer (Bio-Rad, Hercules, CA) and subjected to Ready Gel Zymogram gels (Bio-Rad) under nonreducing condition. After electrophoresis, the gel was renatured in renaturation buffer (Bio-Rad) and developed in development buffer (Bio-Rad). Finally, the gels were stained with $0.5 \%$ Coomassie brilliant blue R-250 and photographed.

\section{Transient transfection}

The siRNAs for MMP9 (MMP9-sil, 5'-CCACCACA ACATCACCTAT-3'; MMP9-si2, 5'-GCATAAGGACGA CGTGAAT- $\left.3^{\prime}\right)$ were designed and synthesized by RiboBio Co. (Guangzhou, China). The siRNAs for FGF1 were purchased from OriGene (SR301569). Transfection was performed using Lipofectamine 3000 (Invitrogen) in accordance with the manufacturer's instructions.

\section{Enzyme linked immunosorbent assay (ELISA)}

The levels of FGF1 in cell culture supernatants were assessed using a human FGF1 ELISA kit (Thermo Fisher Scientific) according to the manufacturer's protocol. 


\section{Bioinformatic analysis}

RNAseqV2 data of 59 pairs of PTC tissues versus paired adjacent noncancerous thyroid tissues and 496 cases of PTC tissues were mined from The Cancer Genome Atlas (TCGA) (https://cancergenome.nih.gov/) using UCSC Xena (http://xena.ucsc.edu/getting-started/).

\section{Statistical analysis}

All statistical analyses were performed using the SPSS17.0 software (SPSS Inc., Chicago, IL). The data were expressed as mean \pm standard deviation (SD). Student $t$ test was performed to compare the differences between two groups. $\mathrm{P}<0.05$ was considered as statistically significant.

\section{Results}

\section{INAVA is upregulated in PTC and associated} with clinicopathologic characteristics of patients

Initially, we assessed the expression of INAVA in PTC. To this end, we analyzed the INAVA expression in 59 pairs of PTC specimens and their corresponding adjacent noncancerous thyroid tissues using thyroid cancer RNAseq data deposited on TCGA. As shown in Fig. 1a, INAVA expression level was upregulated in most (49/59) PTC tissues as compared with their paired adjacent noncancerous thyroid tissues. Next, we collected 16 pairs of PTC and adjacent noncancerous tissues and assessed the expression of INAVA using real time RT-PCR. As shown in Fig. 1b, INAVA expression was elevated in tumor tissues. We further analyzed whether the expression of INAVA is correlated with clinical variables. As shown in Table 1, using RNAseq data deposited on TCGA (496 cases of PTC with clinical information), we found that INAVA expression was significantly associated with $\mathrm{N}$ classification $(P<0.001)$, tumor-node-metastasis (TNM) staging $(P=0.010)$, and $\mathrm{T}$ classification $(P=0.017)$. We further performed IHC to detect the expression of INAVA in 112 cases of PTC specimens. Consistent with the results obtained by analyzing TCGA dataset, the expression level of INAVA in PTC was significantly increased as compared with that in normal thyroid tissues, and also significantly associated with TNM staging $(P=0.007)$ and $\mathrm{N}$ classification $(P=0.005)$ (Table 2$)$. INAVA expression significantly increased in PTC with LN metastasis (LN) as compared with that in PTC with no LN metastasis (NLN) (Fig. 1c, d). Taken together, these data indicate that INAVA expression is elevated in PTC and associated with clinicopathologic features of patients with the disease.

\section{Overexpression of INAVA promotes PTC cells invasion, migration and metastasis}

Given the association of INAVA with more aggressive PTC, we next explored whether the function of INAVA in PTC could explain this association. We ectopically overexpressed INAVA in two PTC cell lines (TPC1 and K1) using empty vector as a control to determine its effect on the aggressive phenotype of the cells (Fig. 2a). As shown in Fig. 2b, c, in vitro cell invasion and migration assays showed that overexpression of INAVA significantly enhanced both the invasion and migration abilities of TPC1 and K1 cells. Scratch wound healing assay was performed to assess the effect of INAVA on cellular migration and the results showed that overexpression of INAVA significantly promoted wound closure in TPC1 and $\mathrm{K} 1$ cells (Fig. 2d). In an in vivo experimental metastasis assay, overexpression of INAVA promoted mouse lung colonization by tail vein-injected K1 cells (Fig. 2e and Additional file 1: Figure S1a).

\section{Knockdown of INAVA inhibits PTC cells invasion, migration and metastasis}

We next established pools of TPC1 and K1 cell lines with stable depletion of INAVA using retroviral-based shRNA vectors (Fig. 3a). As shown in Fig. 3b-e, Transwell assay with or without Matrigel coating showed that silencing of INAVA significantly decreased the invasiveness and migration of the TPC1 and K1 cell lines. Moreover, wound healing assay showed that knockdown of INAVA inhibited the migratory speed of TPC1 and K1 cells in comparison with that of the vector controls (Fig. 3f). In addition, knockdown of INAVA in K1 cells significantly decreased pulmonary metastatic nodule development (Fig. 3g and Additional file 1: Figure S1b). These data, together with results of Fig. 2, suggest that INAVA greatly contributes to the development of PTC invasion and metastasis.

\section{MMP9 is the key molecule mediating INAVA-induced invasion and migration ability of PTC cells}

To explore the molecular mechanisms by which INAVA regulates the invasion and metastasis ability of PTC cells, transcriptome resequencing was performed on INAVAoverexpressing TPC1 cells and vector control cells. Notably, many invasion/metastasis related genes were significantly increased in INAVA overexpressing TPC1 cells as compared with that in vector control (Fig. 4a). Importantly, among these genes, MMP9 was the most elevated gene in response to ectopic overexpression of INAVA. Next, real time RT-PCR assays were performed to confirm the results of deep sequencing analysis. As shown in Fig. 4b, the MMP9 mRNA expression levels were significantly increased and decreased in INAVA overexpressing and knockdown cell lines, respectively, suggesting that transcriptional efficiency of MMP9 is regulated by INAVA in PTC cells. The protein levels of MMP9 in indicated cells were in line with the changes 


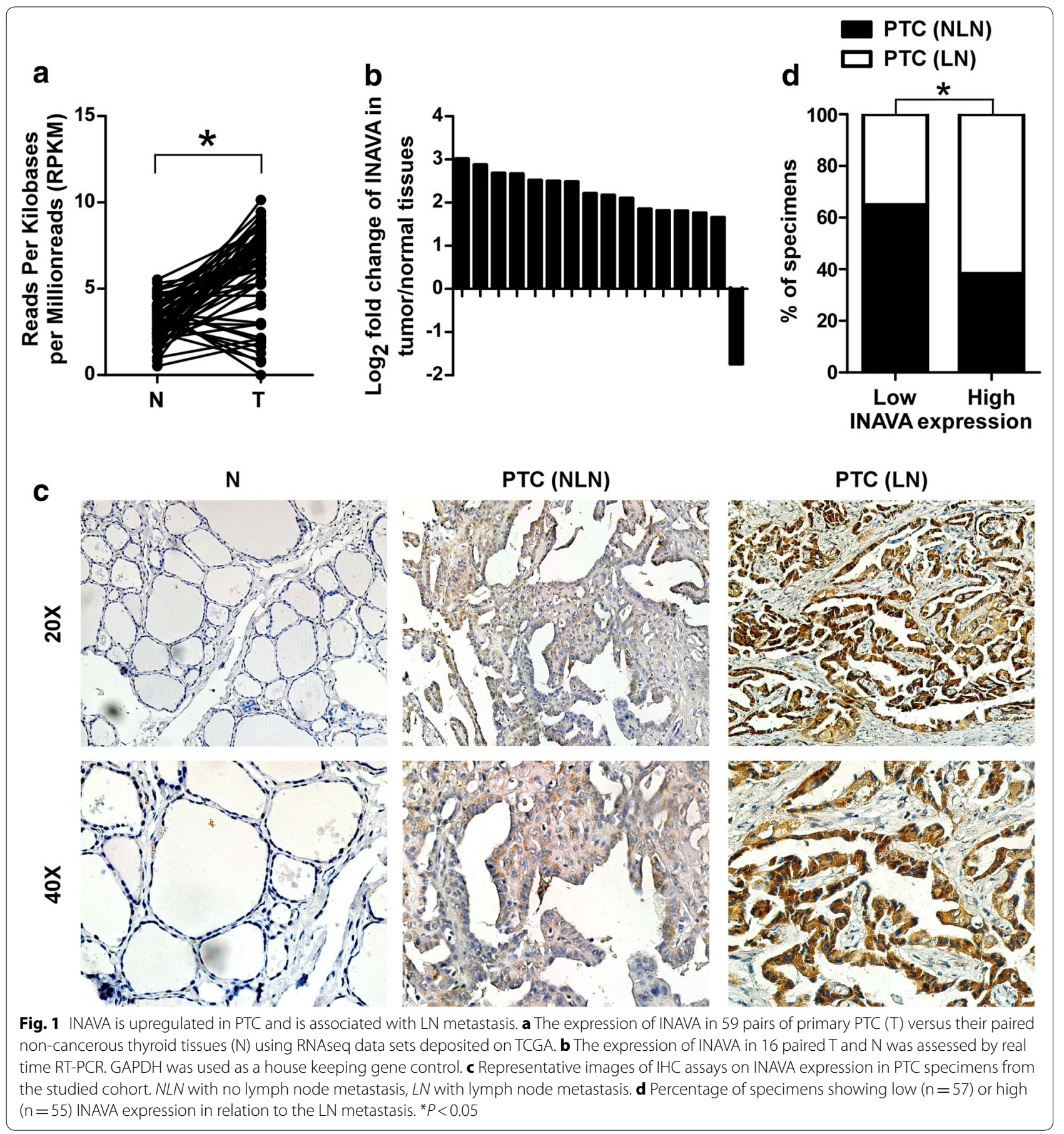

of mRNA (Fig. 4c). As shown in Fig. 4d, the results of gelatin zymography showed that the secretion of MMP9 into culture medium was significantly increased in cells overexpressing INAVA and was significantly decreased in cells with INAVA knockdown. Moreover, knockdown of MMP9 using specific siRNAs in INAVA overexpressing TPC1 and K1 cells (Fig. 4e) suppressed the phenotype caused by INAVA overexpression (Fig. 4f). These results indicate that MMP9 plays a vital role in INAVA-induced cell invasion and migration.

\section{FGF1 mediates the regulation of INAVA on MMP9}

Given that MMP9 has been demonstrated to be regulated by FGF1 [19], together with that the results of deep sequencing analysis showed FGF1 was significantly upregulated in INAVA-overexpressing cells (Fig. 4a), 
Table 1 Association between INAVA expression and clinicopathologic parameters in PTC patients using TCGA dataset

\begin{tabular}{lrrr}
\hline Clinicopathologic variables (n) & \multicolumn{2}{l}{ INAVA } & P value \\
& Low & High & \\
\hline Age (years) & & & \\
$<45$ (331) & 156 & 175 & 0.070 \\
$\geq 45$ (165) & 92 & 73 & \\
Gender & & & \\
Male (131) & 62 & 69 & 0.476 \\
Female (365) & 186 & 179 & \\
TNM stage & & & \\
I and II (329) & 178 & 151 & 0.010 \\
III and IV (165) & 69 & 96 & \\
Unknown (2) & & & \\
T classification & & & \\
T1 and T2 (307) & 167 & 140 & 0.017 \\
T3 and T4 (187) & 81 & 106 & \\
Unknown (2) & & & \\
Lymph node metastasis & & & \\
No (227) & 135 & 92 & $<0.001$ \\
Yes (219) & 83 & 136 & \\
Unknown (50) & & & \\
\hline
\end{tabular}

Table 2 Association between INAVA expression and clinicopathologic parameters in PTC patients

\begin{tabular}{llll}
\hline Clinicopathologic variables ( $\mathbf{n})$ & \multicolumn{2}{l}{ INAVA } & P value \\
\cline { 2 - 3 } & Low & High & \\
\hline Age (years) & & & \\
$\quad<45$ (59) & 32 & 27 & 0.455 \\
$\geq 45$ (53) & 25 & 28 & \\
Gender & & & \\
$\quad$ Male (23) & 12 & 11 & 0.890 \\
Female (89) & 45 & 44 & \\
TNM stage & & & \\
I and II (73) & 44 & 29 & 0.007 \\
III and IV (39) & 13 & 26 & \\
T classification & & & \\
T1 and T2 (69) & 40 & 29 & 0.058 \\
T3 and T4 (43) & 17 & 26 & \\
Lymph node metastasis & & & \\
No (58) & 37 & 21 & 0.005 \\
Yes (54) & 20 & 34 & \\
\hline
\end{tabular}

we hypothesized that FGF1 was involved in the role of INAVA on regulation of MMP9. As expected, the mRNA and protein levels of FGF1 were elevated by overexpression of INAVA but were decreased by depletion of
INAVA in PTC cells (Fig. 5a-d). Consistently, ELISA assays showed that INAVA-overexpressing cells secreted increased amount of FGF1, whereas INAVA silencing reduced the secretion of FGF1 (Fig. 5e). To further confirm the involvement of FGF1 in INAVA-mediated effects, the specific siRNAs were used to knockdown FGF1 in INAVA overexpressing cells. As shown in Fig. 5f, the upregulation of MMP9 induced by INAVA were inhibited by FGF1 silencing. As anticipated, invasive phenotype caused by INAVA overexpression was also suppressed by depletion of FGF1 (Fig. 5g). Taken together, these data indicate FGF1 play a vital role in INAVAinduced MMP9 expression and cell invasion.

\section{INAVA expression correlates with FGF1 and MMP9 expression in primary PTC}

To evaluate the correlations between INAVA expression and the levels of FGF1 and MMP9 in clinical specimens, $\mathrm{IHC}$ analyses were performed. As shown in Fig. $6 \mathrm{a}, \mathrm{b}$, $64.9 \%$ (37 cases) and $61.4 \%$ (35 cases) of samples with low INAVA expression (57 cases), respectively, exhibited low levels of FGF1 and MMP9, whereas 30.9\% (17 cases) and $20.0 \%$ (11 cases) of samples with high INAVA expression (55 cases) showed low expression of FGF1 and MMP9, respectively $(P<0.05)$. Furthermore, we sorted patient cohort into low and high INAVA expression using RNAseq data mined from TCGA (INAVA low and high, $\mathrm{n}=248$ respectively). As shown in Fig. 6c, INAVA expression significantly correlated with the levels of FGF1 and MMP9 in PTC specimens. These results suggest that INAVA overexpression in PTC is associated with FGF1MMP9 axis.

\section{Discussion}

The results of this study provided strong evidence supporting the oncogenic roles of INAVA in PTC. First, the expression of INAVA is significantly elevated in PTC specimens as compared with that in the adjacent nontumorous thyroid specimens. Second, the increased expression of INAVA is associated with clinicopathologic parameters in PTC patients. Third, INAVA promotes the aggressive phenotypes of PTC cells in vitro and in vivo. Fourth, INAVA regulates FGF1-MMP9 axis, thereby contributing to aggressive phenotypes of PTC.

Although the expression of INAVA has been reported to be elevated in lung adenocarcinoma [15], its roles in cancer development and progression are largely unknown. While the molecular mechanism underlying the observed INAVA upregulation in PTC still need to be further investigated, our current data strongly showed that INAVA was significantly upregulated in PTC and was remarkably associated with cervical lymph metastasis by analyzing the TCGA data and our clinical cohorts. 

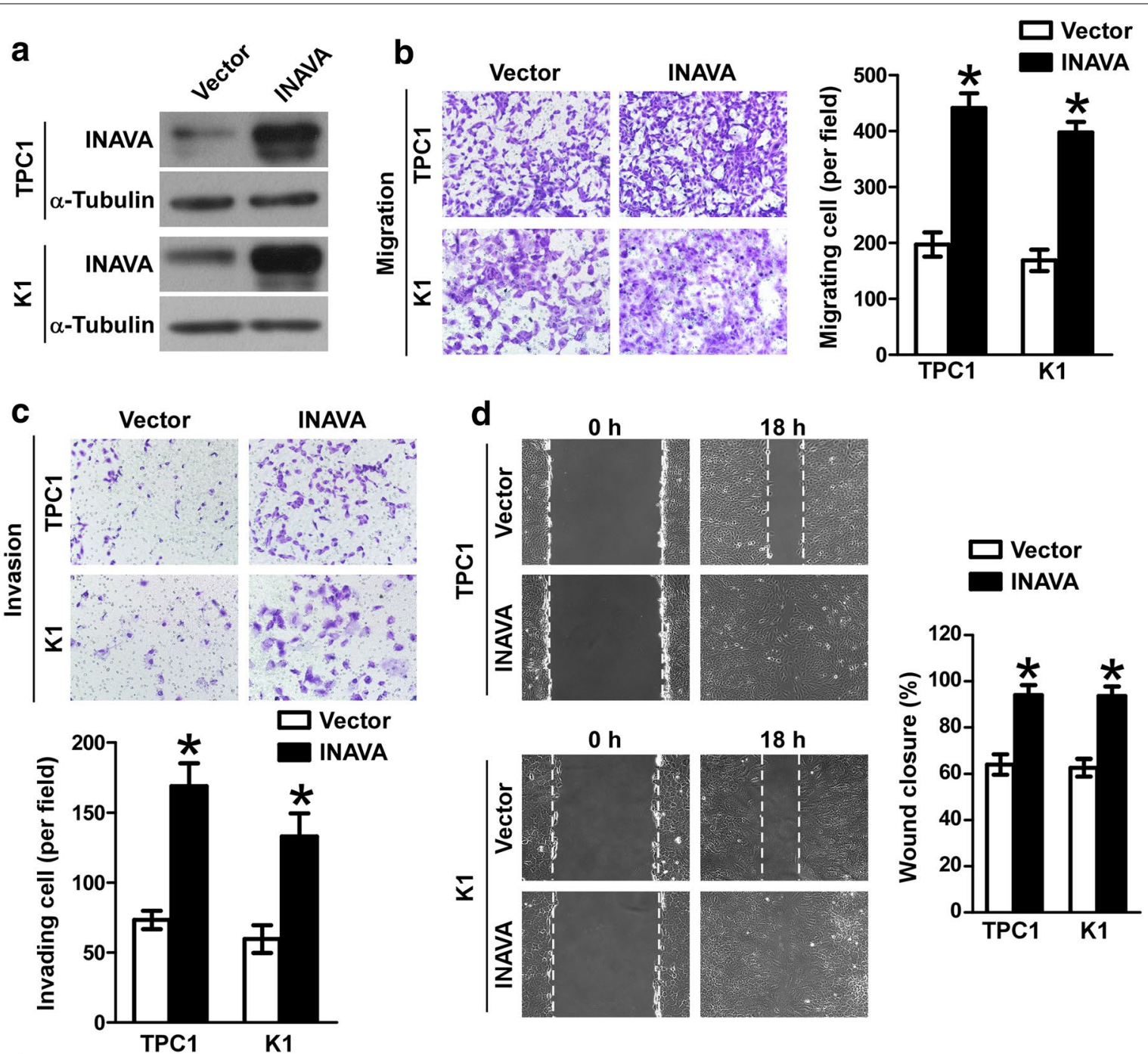

e
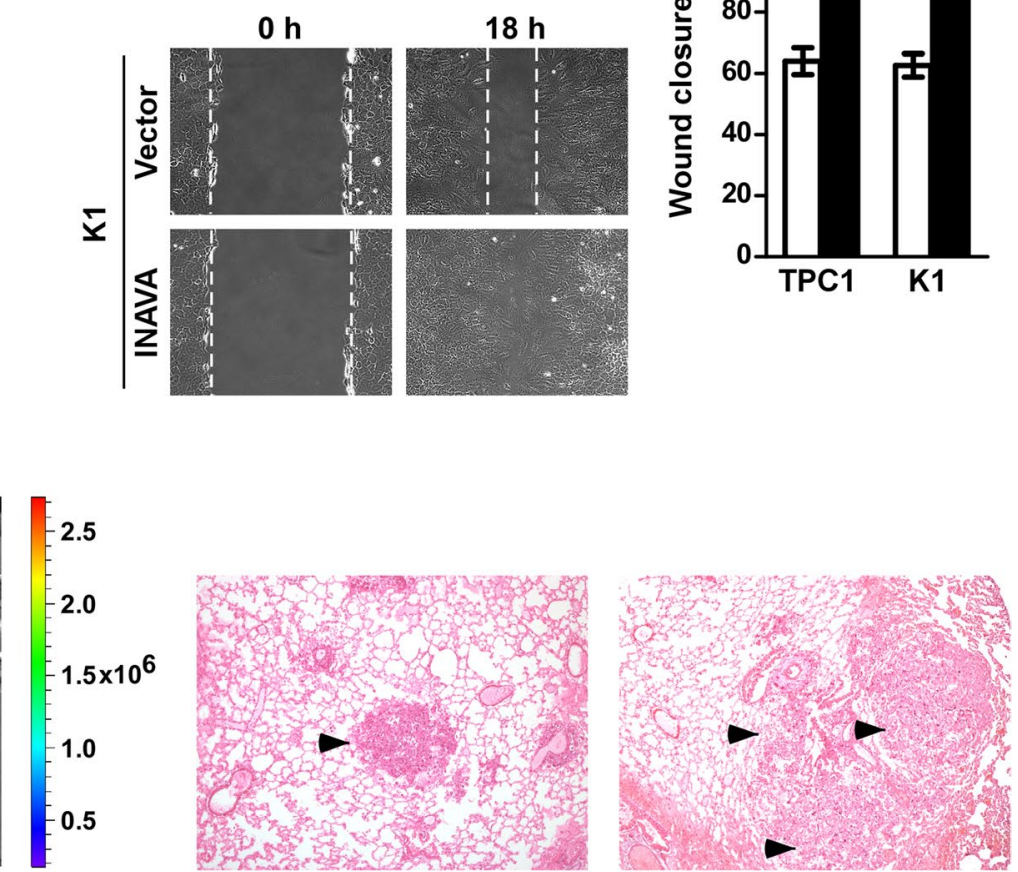

Vector

INAVA $\left(\mathrm{p} / \mathrm{sec} / \mathrm{cm}^{2} / \mathrm{sr}\right)$

Vector

INAVA

K1

\section{K1}

Fig. 2 Overexpression of INAVA promotes cell invasion, migration and metastasis. a Overexpression of INAVA in PTC cell lines (TPC1 and K1) was assessed by WB. a-Tubulin was used as a loading control. b Representative images (left) and quantification (right) of transwell migration assays in TPC1, TPC1-INAVA, K1, and K1-INAVA cells. c Representative images (upper) and quantification (lower) of Transwell invasion assays in indicated cells. d Scratch wound healing assays were performed in TPC1-Vector, TPC1-INAVA, K1-Vector, and K1-INAVA cells (left). Quantification analyses for the wound healing assays were shown (right). e Representative bioluminescence images in mice with tail vein injection of the K1-Vector and K1-INAVA cells (left). Representative histopathology of lung metastasis developed in the indicated animals stained with HE (right). For $\mathbf{b}-\mathbf{d}$, data are quantified as mean \pm SD of 3 independent experiments in the bar graphs. ${ }^{*} P<0.05$ 


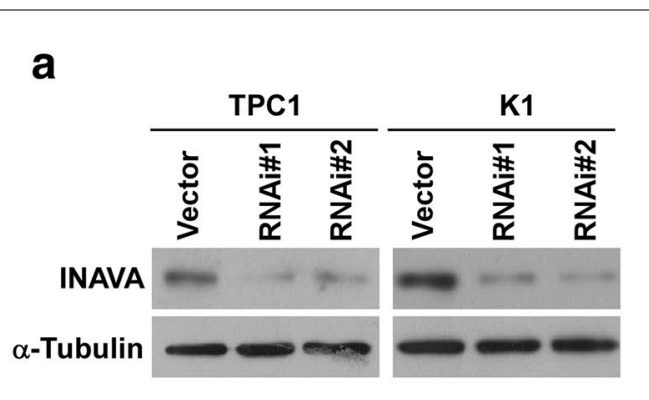

C

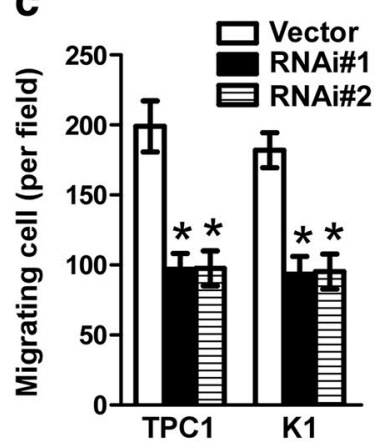

d

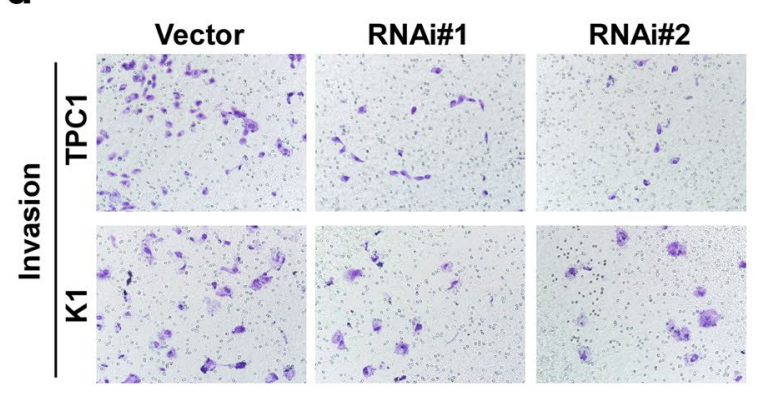

RNAi\#2
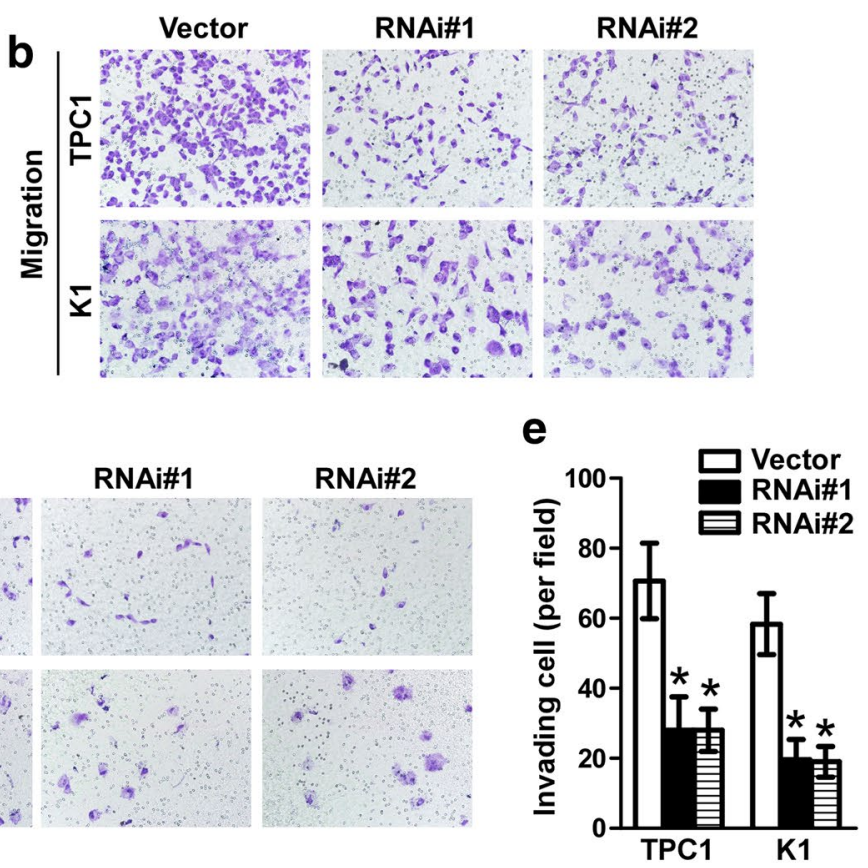

f
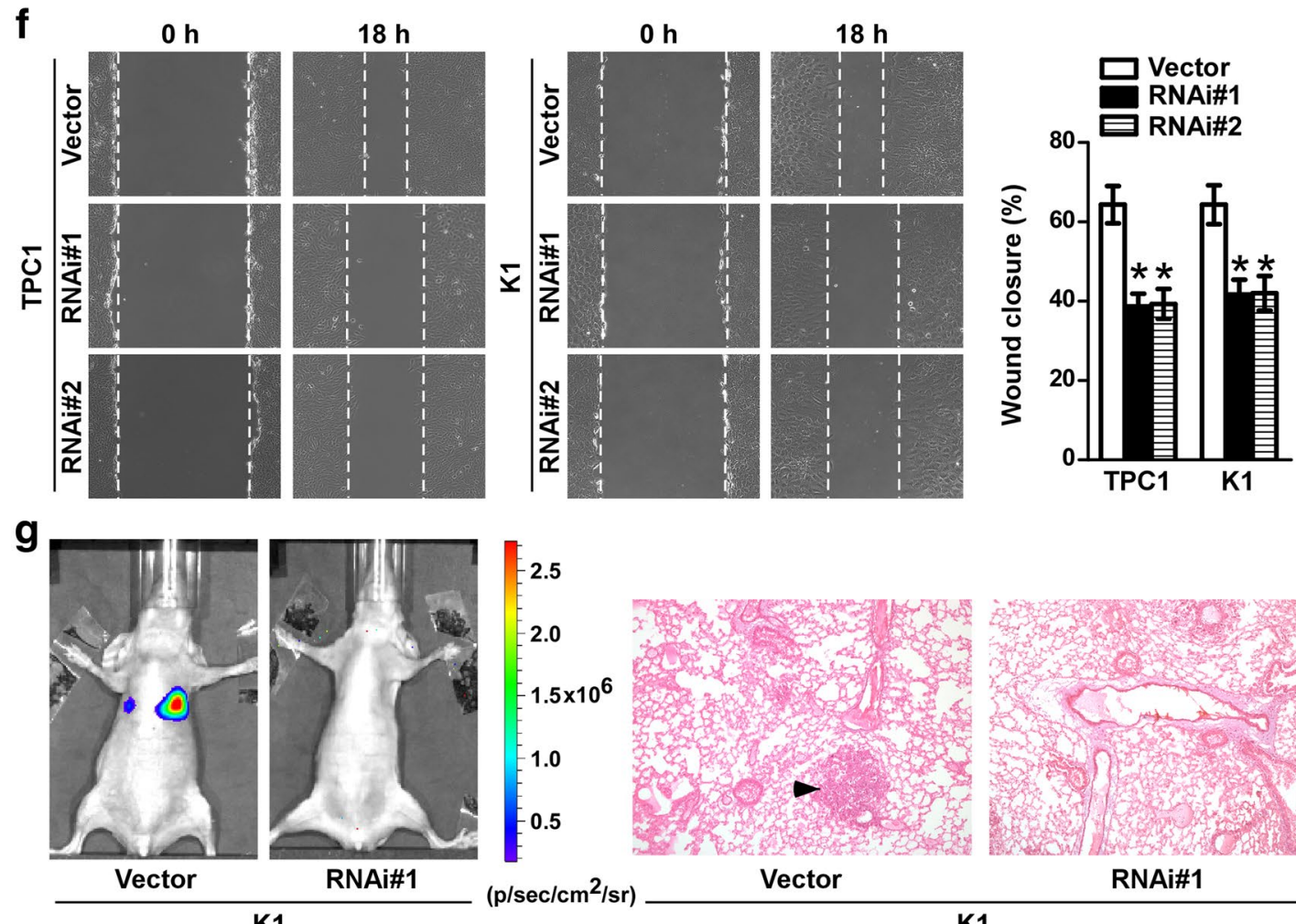

Vector

K1

RNAi\#1

$\left(\mathrm{p} / \mathrm{sec} / \mathrm{cm}^{2} / \mathrm{sr}\right)$

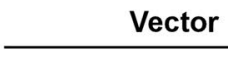

Fig. 3 Silencing of INAVA inhibits cell invasion, migration and metastasis. a Protein expression levels of INAVA in INAVA-silencing and vector-control cells were analyzed by WB. a-Tubulin was used as a loading control. Representative images (b) and quantification (c) of transwell migration assays in indicated cells. Representative images (d) and quantification (e) of transwell invasion assays in indicated cells. $\mathbf{f}$ Scratch wound healing assays were performed in indicated cells (left) and quantification analyses for the assays were shown (right). $\mathbf{g}$ Representative bioluminescence images in mice with tail vein injection of the indicated cells (left) and representative histopathology of lung metastasis stained with HE was shown (right). For b-f, data are quantified as mean \pm SD of 3 independent experiments in the bar graphs. ${ }^{*} P<0.05$ 


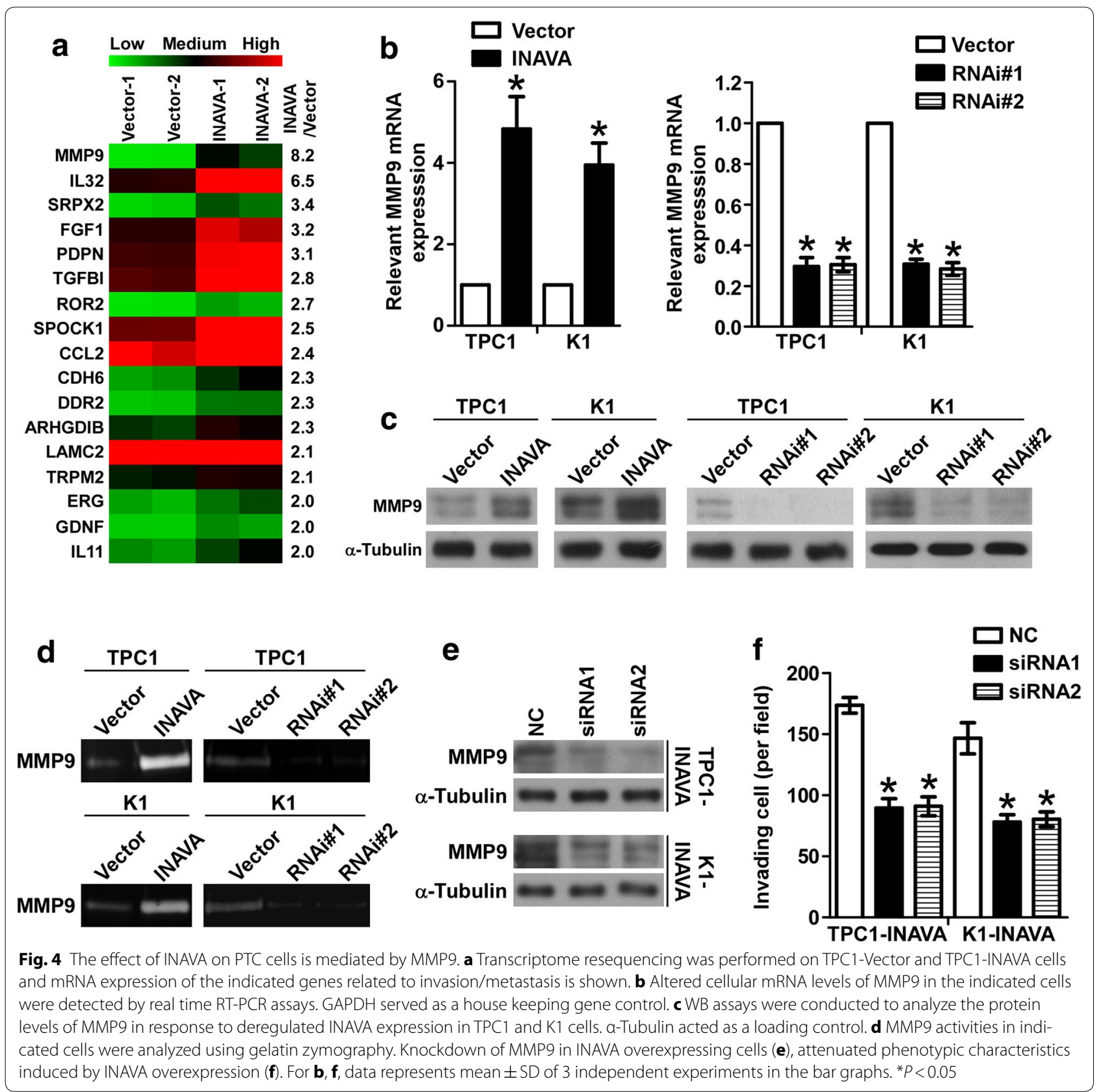

We therefore explored its pro-invasive and pro-metastatic functions in PTC cells by a series of in vitro and in vivo experiments. Our results showed that overexpression of INAVA led to enhancement of the invasive and metastatic capability of TPC1 and K1 cells. On contrary, INAVA silencing in PTC cells showed significantly inhibitory effects on invasion and metastasis. Our data demonstrate that INAVA plays an important role in the progression of malignant disease.
MMP9 degrades type IV collagen and plays important roles in various pathophysiological processes, such as wound healing, tumor invasiveness and progression [20, 21]. It has been demonstrated that MMP9 play important roles in the development and progression of thyroid cancer. For instance, the expression of MMP9 was significantly up-regulated in PTC and was correlated with clinicopathological parameters of the disease [22-24]. MMP9 plays an important role in astrocyte elevated gene-1 induced PTC progression and metastasis [25]. 


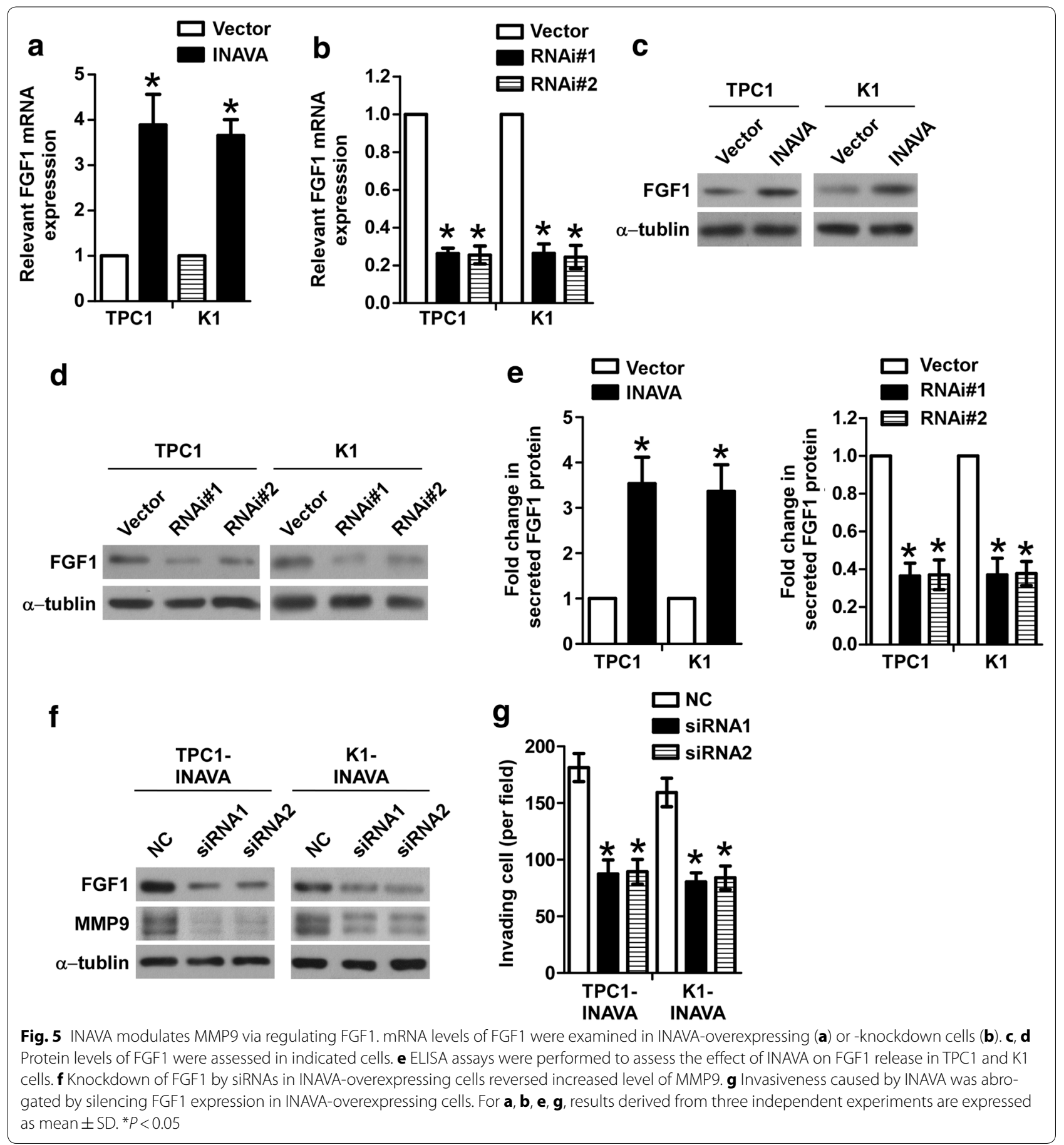

Further studies that aim at investigating the regulatory mechanisms underlying the MMP9 in PTC will improve our understanding of etiology of the disease. One of the key finding of the current study is represented by the identification of a new signaling cascade in PTC cells. Elevated INAVA upregulates the expression and secretion of FGF1, which subsequently results in upregulation of MMP9 and aggressive phenotypes of PTC cells. The data derived from current mechanistic study provide an explanation for the observed association between INAVA overexpression and cervical lymph metastasis in PTC. Of note, it would also be interesting to explore whether the same chain reaction also presents in other cancer types with INAVA overexpression. 


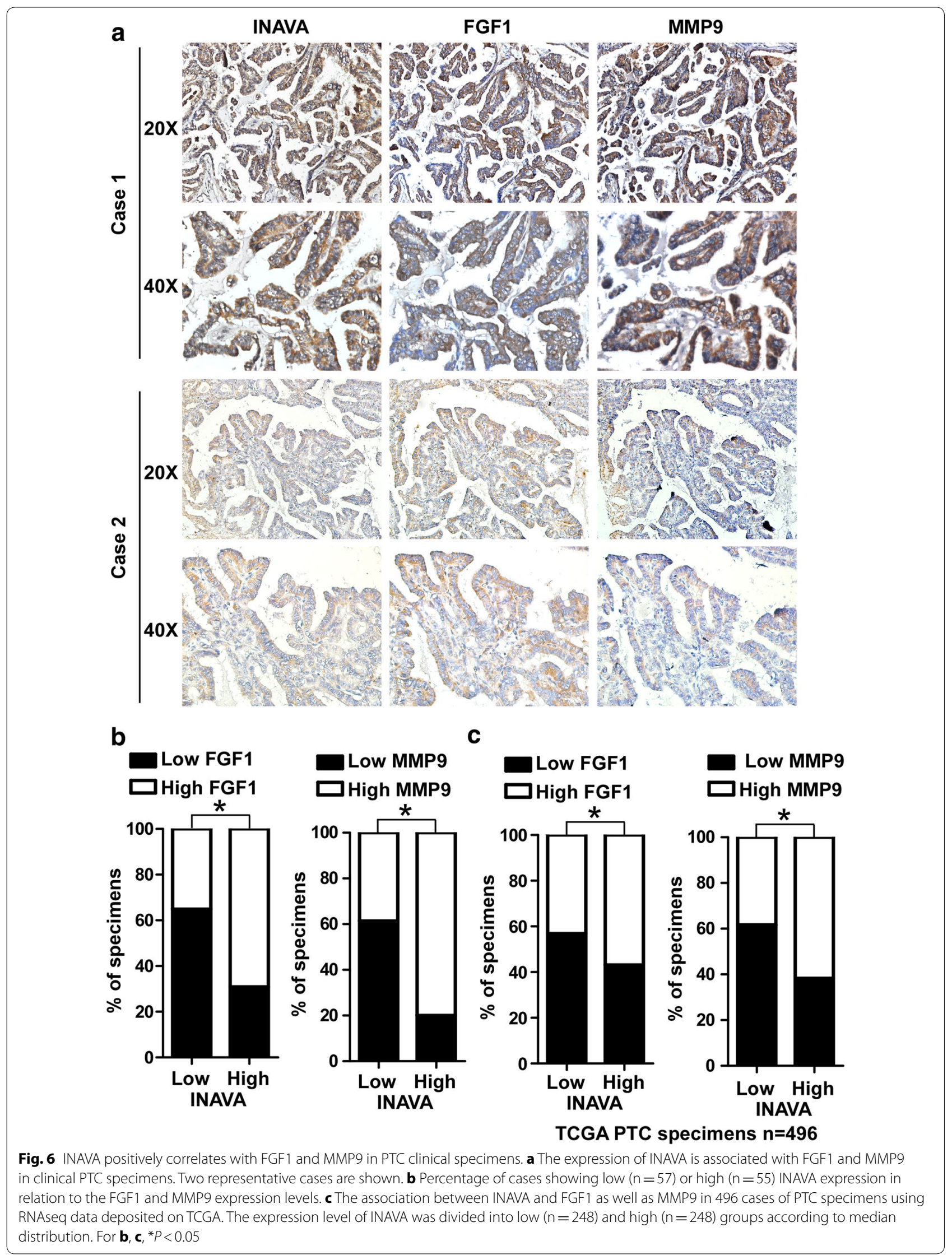


Although most thyroid cancers of follicular epithelial derivation are well differentiated and well behaved, a small subset of thyroid cancers behave aggressively via genetic and epigenetic alterations [26-28]. PTC is known for its early spread to regional lymph nodes and growing evidence has demonstrated that central lymph node metastasis is adversely associated with survival $[29,30]$. Distant metastasis occurs in only $<1 \%$ of thyroid cancer, but strongly associates with poor clinical outcomes [3133]. Delineating the molecular aspects of the LN metastasis and distant metastasis in PTC will lead to a better understanding of the disease. Our finding provides new insights into the molecular mechanisms underlying the aggressive behavior of PTC by identifying INAVA as a novel promoter of tumor cell invasion and metastasis.

FGF1, an oncogene, has been demonstrated to regulate many cellular processes, including cell invasion, proliferation, and survival [34, 35]. FGF1 is upregulated in various tumors and is involved in progression of malignances [36-38]. The elevated expression of FGF1 has been found in PTC [39]. To delineate the regulatory mechanisms of deregulation of FGF1 in cancers is of great interest. Our data obtained in vitro using TPC1 and K1 cell lines showed that overexpression of INAVA enhanced the expression and secretion of FGF1, whereas INAVA silencing inhibited FGF1 expression and secretion. In addition, our clinical findings demonstrated a strong correlation between INAVA and FGF1 expression. Our data provide new insights into the current understanding on the regulatory networks that control the expression of FGF1 in malignant diseases.

FGF1 can be released into the extracellular environment via unclear mechanisms, by doing this, it acts as a paracrine or autocrine factor to activate specific cell surface receptors [40]. There are two possible sources of FGF1 within epithelial cancers, namely, the cancer cells or surrounding stromal cells. FGF1 may be secreted from either of these two sources and acts in a paracrine and/ or autocrine manner [41]. Secreted FGF1 exerts its biological function via binding to, and activation of, fibroblast growth factor receptors (FGFRs) with intrinsic tyrosine kinase activity at the cellular surface and thereby triggering intracellular signaling cascades, including mitogen-activated protein kinase (MAPK), nuclear factor-kappa B (NF-kB), and phospholipase CY (PLCY) signaling $[19,42,43]$. Of specific note, MAPK and NF-kB pathways are well known for regulating the expression of MMP9 [44, 45]. In current study, we found that INAVA increased the expression as well as the secretion of FGF1 in PTC cells. It is likely that MAPK and NF- $\mathrm{KB}$ pathways might be involved in INAVA-induced MMP9 expression. Another interesting issue need to be further investigated is whether the FGF1 secreted by the tumor cell may act in a paracrine manner to affect the surrounding stromal cells, which in turn facilitates the development and progression of PTC.

\section{Conclusion}

In summary, our data indicate that INAVA expression is elevated in PTC. Our data also show that INAVA expression is associated with aggressive phenotypes of PTC. We demonstrate that INAVA overexpression enhances invasion and metastasis via upregulation of MMP9. In addition, shRNA-mediated knockdown experiments further confirm the pro-invasive and pro-metastatic roles of INAVA in PTC. Furthermore, we show that the upregulation of MMP9 induced by INAVA is mediated by elevated FGF1. Taken as a whole, our data reveal a novel molecular mechanism that involves the aggressive phenotypes of PTC and may prove clinically useful for developing a new therapeutic target for PTC invasion and metastasis.

\section{Additional file}

Additional file 1: Figure S1. INAVA regulates PTC cell metastasis. Metastasis area was calculated using Image J software in HE stained sections.

\section{Abbreviations}

ALOX5: arachidonate 5-lipoxygenase; BCA: bicinchoninic acid assay; DMEM: Dulbecco's modified Eagle's medium; ECACC: European Collection of Cell Cultures; ECM: extracellular matrix; ECL: enhanced chemiluminescence; ELISA: enzyme linked immunosorbent assay; FGF1: fibroblast growth factor 1; GAPDH: glyceraldehyde-3-phosphate dehydrogenase; GEO: gene expression omnibus; HE: hematoxylin and eosin; IHC: immunohistochemistry; INAVA: innate immunity activator; LN: lymph node; NLN: with no lymph node metastasis; MMPs: matrix metalloproteinases; PTC: papillary thyroid cancer; RT-PCR: reverse transcription-polymerase chain reaction; SDS-PAGE: sodium dodecyl sulfate-polyacrylamide gel electrophoresis; STR: short tandem repeat; TCGA: The Cancer Genome Atlas; TNM: tumor-node-metastasis; WB: western blotting.

\section{Authors' contributions}

$H G, Y G$, and LL planned and conducted experiments, analyzed the data and wrote the text. RY, WL, and HL conducted experiments and analyzed data. HX participated in its design and contributed to discuss the data and text. YL conceived of the study, and participated in its design and coordination and critical review of the manuscript. All authors read and approved the final manuscript.

\section{Author details}

${ }^{1}$ Department of Endocrinology and Diabetes Center, The First Affiliated Hospital of Sun Yat-sen University, 58 Zhongshan Road II, Guangzhou 510080, Guangdong, China. ${ }^{2}$ Department of Thyroid and Breast Surgery, The First Affiliated Hospital of Sun Yat-sen University, Guangzhou, China.

\section{Acknowledgements}

We thank Prof. Haixia Guan (China Medical University, China) for TPC-1 cell line.

\section{Competing interests}

The authors declare that they have no competing interests.

\section{Availability of data and materials}

RNAseqV2 data of 59 pairs of PTC tissues versus paired adjacent noncancerous thyroid tissues and 496 cases of PTC tissues were mined from The Cancer Genome Atlas (TCGA) (https://cancergenome.nih.gov/) using UCSC Xena (http://xena.ucsc.edu/getting-started/). The transcriptome resequencing data described herein have been deposited in the Gene Expression Omnibus 
(GEO) database and are accessible through GEO series Accession Number GSE100392.

\section{Consent for publication}

Not applicable.

\section{Ethics approval and consent to participate}

The use of clinical specimens and clinical data were reviewed and approved by the Institutional Research Ethics Committee of the First Affiliated Hospital of Sun Yat-sen University. Written informed consent was obtained from all patients who participated in the study. Animal experiments were approved by the Institutional Animal Care and Use Committee of the First Affiliated Hospital, Sun Yat-sen University.

\section{Funding}

This research was supported by Grants from the National Natural Science Foundation of China (Nos. 81572624, 81572623, 81602347) and the Natural Science Foundation of Guangdong Province (No. 2016A030310169).

\section{Publisher's Note}

Springer Nature remains neutral with regard to jurisdictional claims in published maps and institutional affiliations.

Received: 14 December 2017 Accepted: 30 March 2018 Published online: 05 April 2018

\section{References}

1. Fagin JA, Wells SA Jr. Biologic and clinical perspectives on thyroid cancer. N Engl J Med. 2016;375(23):2307.

2. Nikiforov YE. Molecular analysis of thyroid tumors. Mod Pathol. 2011;24(Suppl 2):S34-43.

3. Torregrossa L, Viola D, Sensi E, Giordano M, Piaggi P, Romei C, Materazzi G, Miccoli P, Elisei R, Basolo F. Papillary thyroid carcinoma with rare Exon 15 BRAF mutation has indolent behavior: a single-institution experience. J Clin Endocrinol Metab. 2016;101(11):4413-20.

4. Randolph GW, Duh QY, Heller KS, LiVolsi VA, Mandel SJ, Steward DL, Tufano RP, Tuttle RM, American Thyroid Association Surgical Affairs Committee's Taskforce on Thyroid Cancer Nodal S. The prognostic significance of nodal metastases from papillary thyroid carcinoma can be stratified based on the size and number of metastatic lymph nodes, as well as the presence of extranodal extension. Thyroid. 2012;22(11):1144-52.

5. Leboulleux S, Rubino C, Baudin E, Caillou B, Hartl DM, Bidart JM, Travagli JP, Schlumberger M. Prognostic factors for persistent or recurrent disease of papillary thyroid carcinoma with neck lymph node metastases and/ or tumor extension beyond the thyroid capsule at initial diagnosis. J Clin Endocrinol Metab. 2005;90(10):5723-9.

6. Bravo-Cordero JJ, Hodgson L, Condeelis J. Directed cell invasion and migration during metastasis. Curr Opin Cell Biol. 2012;24(2):277-83.

7. Kessenbrock K, Plaks V, Werb Z. Matrix metalloproteinases: regulators of the tumor microenvironment. Cell. 2010;141(1):52-67.

8. Egeblad M, Werb Z. New functions for the matrix metalloproteinases in cancer progression. Nat Rev Cancer. 2002;2(3):161-74.

9. McCawley LJ, Matrisian LM. Matrix metalloproteinases: they're not just for matrix anymore! Curr Opin Cell Biol. 2001;13(5):534-40.

10. Nannuru KC, Futakuchi M, Varney ML, Vincent TM, Marcusson EG, Singh RK. Matrix metalloproteinase (MMP)-13 regulates mammary tumor-induced osteolysis by activating MMP9 and transforming growth factor-beta signaling at the tumor-bone interface. Cancer Res. 2010;70(9):3494-504.

11. Ahmed M, Uddin S, Hussain AR, Alyan A, Jehan Z, Al-Dayel F, Al-Nuaim A, Al-Sobhi S, Amin T, Bavi P, et al. FoxM1 and its association with matrix metalloproteinases (MMP) signaling pathway in papillary thyroid carcinoma. J Clin Endocrinol Metab. 2012;97(1):E1-13.

12. Kummer NT, Nowicki TS, Azzi JP, Reyes I, lacob C, Xie S, Swati I, Darzynkiewicz Z, Gotlinger KH, Suslina N, et al. Arachidonate 5 lipoxygenase expression in papillary thyroid carcinoma promotes invasion via MMP-9 induction. J Cell Biochem. 2012;113(6):1998-2008.
13. Liu Y, Xu H, Chen S, Chen X, Zhang Z, Zhu Z, Qin X, Hu L, Zhu J, Zhao $G P$, et al. Genome-wide interaction-based association analysis identified multiple new susceptibility Loci for common diseases. PLoS Genet. 2011;7(3):e1001338.

14. Franke A, McGovern DP, Barrett JC, Wang K, Radford-Smith GL, Ahmad T, Lees CW, Balschun T, Lee J, Roberts R, et al. Genome-wide meta-analysis increases to 71 the number of confirmed Crohn's disease susceptibility loci. Nat Genet. 2010;42(12):1118-25.

15. Zhang $Y$, Wang $H$, Wang J, Bao L, Wang L, Huo J, Wang X. Global analysis of chromosome 1 genes among patients with lung adenocarcinoma, squamous carcinoma, large-cell carcinoma, small-cell carcinoma, or noncancer. Cancer Metastasis Rev. 2015;34(2):249-64.

16. Guan H, Wei G, Wu J, Fang D, Liao Z, Xiao H, Li M, Li Y. Down-regulation of miR-218-2 and its host gene SLIT3 cooperate to promote invasion and progression of thyroid cancer. J Clin Endocrinol Metab. 2013;98(8):E1334-44.

17. Bouvet M, Tsuji K, Yang M, Jiang P, Moossa AR, Hoffman RM. In vivo colorcoded imaging of the interaction of colon cancer cells and splenocytes in the formation of liver metastases. Cancer Res. 2006;66(23):11293-7.

18. Scarpino S, Duranti E, Giglio S, Di Napoli A, Galafate D, Del Bufalo D, Desideri M, Socciarelli F, Stoppacciaro A, Ruco L. Papillary carcinoma of the thyroid: high expression of COX-2 and low expression of KAI-1/ CD82 are associated with increased tumor invasiveness. Thyroid. 2013;23(9):1127-37.

19. Lungu G, Covaleda L, Mendes O, Martini-Stoica H, Stoica G. FGF-1-induced matrix metalloproteinase- 9 expression in breast cancer cells is mediated by increased activities of NF-kappaB and activating protein-1. Mol Carcinog. 2008;47(6):424-35.

20. Annahazi A, Abraham S, Farkas K, Rosztoczy A, Inczefi O, Foldesi I, Szucs M, Rutka M, Theodorou V, Eutamene $H$, et al. A pilot study on faecal MMP9: a new noninvasive diagnostic marker of colorectal cancer. Br J Cancer. 2016;114(7):787-92.

21. Chang JW, Kang SU, Shin YS, Kim KI, Seo SJ, Yang SS, Lee JS, Moon E, Lee K, Kim CH. Non-thermal atmospheric pressure plasma inhibits thyroid papillary cancer cell invasion via cytoskeletal modulation, altered MMP-2/-9/ uPA activity. PLoS ONE. 2014;9(3):e92198.

22. Meng XY, Zhang Q, Li Q, Lin S, Li J. Immunohistochemical levels of cyclo-oxygenase-2, matrix metalloproteinase-9 and vascular endothelial growth factor in papillary thyroid carcinoma and their clinicopathological correlations. J Int Med Res. 2014;42(3):619-27.

23. Wang $N$, Jiang $R$, Yang JY, Tang C, Yang L, Xu M, Jiang QF, Liu ZM. Expression of TGF-beta1, SNAI1 and MMP-9 is associated with lymph node metastasis in papillary thyroid carcinoma. J Mol Histol. 2014;45(4):391-9.

24. Marecko I, Cvejic D, Selemetjev S, Paskas S, Tatic S, Paunovic I, Savin S. Enhanced activation of matrix metalloproteinase-9 correlates with the degree of papillary thyroid carcinoma infiltration. Croat Med J. 2014;55(2):128-37.

25. Huang LL, Wang Z, Cao CJ, Ke ZF, Wang F, Wang R, Luo CQ, Lu X, Wang LT. AEG-1 associates with metastasis in papillary thyroid cancer through upregulation of MMP2/9. Int J Oncol. 2017;51(3):812-22.

26. Carling T, Udelsman R. Thyroid cancer. Annu Rev Med. 2014;65:125-37.

27. Tavares C, Coelho MJ, Melo M, da Rocha AG, Pestana A, Batista R, Salgado C, Eloy C, Ferreira L, Rios E, et al. pmTOR is a marker of aggressiveness in papillary thyroid carcinomas. Surgery. 2016;160(6):1582-90.

28. Chou CK, Chi SY, Huang CH, Chou FF, Huang CC, Liu RT, Kang HY. IRAK1, a target of miR-146b, reduces cell aggressiveness of human papillary thyroid carcinoma. J Clin Endocrinol Metab. 2016;101(11):4357-66.

29. Cho SY, Lee TH, Ku YH, Kim HI, Lee GH, Kim MJ. Central lymph node metastasis in papillary thyroid microcarcinoma can be stratified according to the number, the size of metastatic foci, and the presence of desmoplasia. Surgery. 2015;157(1):111-8.

30. Kim SK, Park I, Woo JW, Lee JH, Choe JH, Kim JH, Kim JS. Predictive factors for lymph node metastasis in papillary thyroid microcarcinoma. Ann Surg Oncol. 2016;23(9):2866-73.

31. Furlan JC, Bedard YC, Rosen IB. Clinicopathologic significance of histologic vascular invasion in papillary and follicular thyroid carcinomas. J Am Coll Surg. 2004;198(3):341-8.

32. Thomas L, Lai SY, Dong W, Feng L, Dadu R, Regone RM, Cabanillas ME. Sorafenib in metastatic thyroid cancer: a systematic review. Oncologist. 2014;19(3):251-8. 
33. Choi YM, Kim WG, Kwon H, Jeon MJ, Lee JJ, Ryu JS, Hong EG, Kim TY, Shong YK, Kim WB. Early prognostic factors at the time of diagnosis of bone metastasis in patients with bone metastases of differentiated thyroid carcinoma. Eur J Endocrinol. 2016;175(3):165-72.

34. Wiedlocha A, Sorensen V. Signaling, internalization, and intracelIular activity of fibroblast growth factor. Curr Top Microbiol Immunol. 2004;286:45-79.

35. Huang Z, Tan Y, Gu J, Liu Y, Song L, Niu J, Zhao L, Srinivasan L, Lin Q, Deng J, et al. Uncoupling the mitogenic and metabolic functions of FGF1 by tuning FGF1-FGF receptor dimer stability. Cell Rep. 2017;20(7):1717-28.

36. Pirou C, Montazer-Torbati F, Jah N, Delmas E, Lasbleiz C, Mignotte B, Renaud F. FGF1 protects neuroblastoma SH-SY5Y cells from p53-dependent apoptosis through an intracrine pathway regulated by FGF1 phosphorylation. Cell Death Dis. 2017;8(8):e3023.

37. Szlachcic A, Zakrzewska M, Lobocki M, Jakimowicz P, Otlewski J. Design and characteristics of cytotoxic fibroblast growth factor 1 conjugate for fibroblast growth factor receptor-targeted cancer therapy. Drug Des Devel Ther. 2016:10:2547-60.

38. Jiao J, Zhao X, Liang Y, Tang D, Pan C. FGF1-FGFR1 axis promotes tongue squamous cell carcinoma (TSCC) metastasis through epithelial-mesenchymal transition (EMT). Biochem Biophys Res Commun. 2015;466(3):327-32

39. Eggo MC, Hopkins JM, Franklyn JA, Johnson GD, Sanders DS, Sheppard MC. Expression of fibroblast growth factors in thyroid cancer. J Clin Endocrinol Metab. 1995;80(3):1006-11.
40. Powers CJ, McLeskey SW, Wellstein A. Fibroblast growth factors, their receptors and signaling. Endocr Relat Cancer. 2000;7(3):165-97.

41. Nies VJ, Sancar G, Liu W, van Zutphen T, Struik D, Yu RT, Atkins AR, Evans RM, Jonker JW, Downes MR. Fibroblast growth factor signaling in metabolic regulation. Front Endocrinol (Lausanne). 2016;19(6):193.

42. Takahashi M, Okubo N, Chosa N, Takahashi N, Ibi M, Kamo M, Mizuki $\mathrm{H}$, Ishisaki A, Kyakumoto S. Fibroblast growth factor-1-induced ERK1/2 signaling reciprocally regulates proliferation and smooth muscle cell differentiation of ligament-derived endothelial progenitor cell-like cells. Int J Mol Med. 2012;29(3):357-64.

43. Browaeys-Poly E, Perdereau D, Lescuyer A, Burnol AF, Cailliau K. Akt interaction with $\mathrm{PLC}($ gamma) regulates the $\mathrm{G}(2) / \mathrm{M}$ transition triggered by FGF receptors from MDA-MB-231 breast cancer cells. Anticancer Res. 2009;29(12):4965-9.

44. Wei Y, Zhao L, He W, Yang J, Geng C, Chen Y, Liu T, Chen H, Li Y. Benzo[a] pyrene promotes gastric cancer cell proliferation and metastasis likely through the Aryl hydrocarbon receptor and ERK-dependent induction of MMP9 and c-myc. Int J Oncol. 2016;49(5):2055-63.

45. Zhao G, Wu H, Jiang K, Chen X, Wang X, Qiu C, Guo M, Deng G. The anti-inflammatory effects of interferon tau by suppressing NF-KB/MMP9 in macrophages stimulated with Staphylococcus aureus. J Interferon Cytokine Res. 2016;36(8):516-24
Ready to submit your research? Choose BMC and benefit from:

- fast, convenient online submission

- thorough peer review by experienced researchers in your field

- rapid publication on acceptance

- support for research data, including large and complex data types

- gold Open Access which fosters wider collaboration and increased citations

- maximum visibility for your research: over 100M website views per year

At BMC, research is always in progress.

Learn more biomedcentral.com/submissions 